\title{
INVESTIGATIONS OF THE DYNAMICS OF A BISTABLE ELECTROMAGNET WITH IMPROVED CHARACTERISTICS FOR MEDIUM VOLTAGE VACUUM CIRCUIT BREAKERS
}

Introduction. Currently, for switching medium voltage circuits, vacuum circuit breakers are widely used, which have good arcing properties and high breaking capacity. One of the problems of creating the drive mechanism of such apparatus is the need to ensure the absence of contact welding when a through current of a short circuit of a given duration flows through them, which is achieved due to a certain amount of contact pressure. One of the problems arising in the design of circuit breakers is the need to fix the mechanism with a mechanical lock, which should hold the mechanism securely. This leads to significant specific mechanical loads, which in turn reduces the reliability of the circuit breaker. One way to solve these problems is to create a drive based on monostable or bistable electromagnetic actuators with highly coercive permanent magnets, which provide reliable fixation of the position of the contacts. Purpose. Investigation of the improved design of a bistable electromagnetic actuator based on permanent magnets of a medium voltage vacuum circuit breaker. Methods. Theoretical and experimental research and comparative analysis of existing and developed electromagnetic actuators. Conclusions. A new design of an electromagnetic bistable actuator with reduced overall dimensions is developed and tested. The electromechanical characteristics of the actuator correspond to the technical specifications, which is confirmed by both theoretical and experimental studies. The proposed actuator can be used as a drive mechanism for medium voltage vacuum circuit breakers. References 10, figures 17, tables 3 . Key words: bistable electromagnet, actuator, vacuum circuit breaker, multiphysics model.

В статье исследован новый бистабильный электромагнит с высококоэрцитивными постоянными магнитами, который предполагается использовать в качестве актуатора вакуумных выключателей средних напряэений. Приводится теоретическое и экспериментальное исследование усовершенствованной конструкции электромагнита с целью сравнительного анализа его параметров с параметрами имеющейся конструкции. Теоретическое исследование базируется на мультифизической модели, которая включает расчет статического и динамического электромагнитных полей в нелинейной проводящей неоднородной среде с учетом постоянных магнитов, нелинейных уравнений разрядной цепи накопительного конденсатора, нелинейных уравнений движения. Экспериментальные исследования, которые проводились на реальном вакуумном выключателе, показали соответствие параметров нового бистабильного электромагнита расчетным показателям. Направление дальнейших исследований представляются в виде оптимизации геометрии электромагнита и схемы управления. Библ. 10, рис. 17, табл. 3.

Ключевые слова: бистабильный электромагнит, актуатор, вакуумный выключатель, мультифизическая модель.

Introduction. In recent years, vacuum switching devices having unique arcing properties and high breaking capacity [1] are widely used in medium voltage circuits. One of the most important characteristics of such apparatus is the rated short-time withstand current - the current that the circuit breaker must withstand for given time in accordance with the test conditions [2] defined by the standard, which is achieved due to a certain value of contact pressure. Under the electromagnetic actuator we understand the device that creates the movement of contacts when excited by an electric signal of an electromagnetic field in an electromagnet. Here, the movement of the contacts in one direction is provided by the traction force of the electromagnet, and in the other one by contact and disconnecting springs. Thus, the electromagnet together with the contact springs forms an electromagnetic actuator. From experimental data it is known $[1,3]$ that to ensure the breaking capacity of the circuit breaker of $20 \mathrm{kA}$, contact pressure should be of the order $2 \mathrm{kN}$ per pole or about $6 \mathrm{kN}$ per three poles, respectively. Such contact pressures are provided by Belleville springs, therefore, the mechanical opposing characteristic has its own peculiarity (Fig. 1), which consists in a jump in the opposing force at the moment of contact touch. This leads to a slowdown in the velocity of movement of the armature of the electromagnet and a possible «freezing» of the drive mechanism [4] (if the kinetic energy of the moving parts is not enough to overcome the area where the electromagnetic force is less than the opposing one).

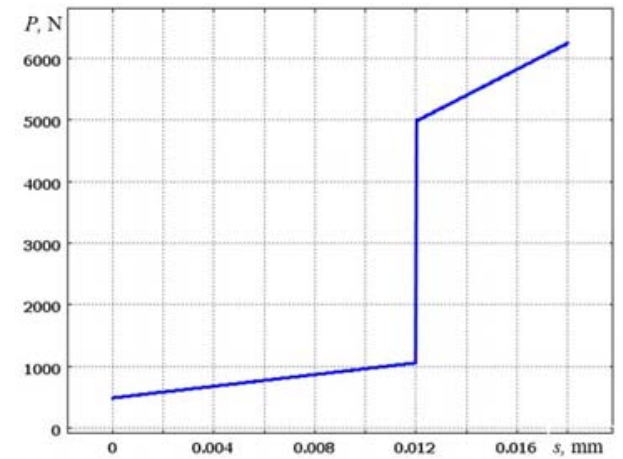

Fig. 1. The dependence of the opposing force $(P)$ as a function of the stroke $(s)$ of the armature

There are publications on the design and calculation of electromagnetic actuators [4-6]. One of the problems arising in the design of circuit breakers is the need to fix the mechanism of the circuit breaker in extreme positions. Actuators of vacuum circuit breakers, as a rule, use neutral electromagnets or electric motors, the main disadvantage of which is the presence of mechanical locks - mechanical latches that securely hold the mechanism in the on position. The main requirement for mechanical latches is the small force required to release the free trip mechanism when the circuit breaker is switched off, which is associated with its velocity. This leads to significant specific mechanical loads on the mechanical element, which in turn reduces the reliability of the circuit

(C) E.I. Baida, B.V. Klymenko, S.V. Vyrovets, M.G. Pantelyat, M. Clemens 
breaker. In addition, electromagnetic and spring-motor actuators are complex and require qualified service.

One way to solve these problems is to create an actuator based on monostable (Fig. 2,a) or bistable (Fig. 2,b) polarized electromagnets with highly coercive permanent magnets, which provide reliable fixation of contacts in extreme positions, consuming energy only in transient modes. The advantage of such electromagnets is the simplicity of design and reliability.

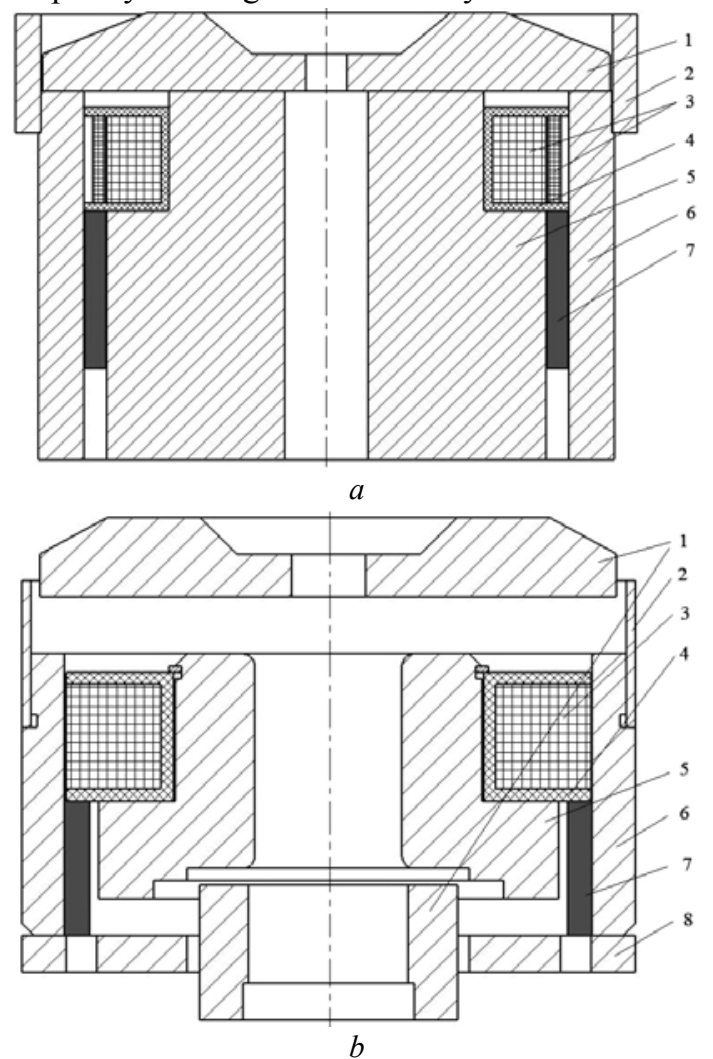

Fig. 2. Sketches of monostable $(a)$ and bistable $(b)$ electromagnets

The designs of shown in Fig. 2 electromagnets are similar. Each includes a movable armature (1), a ferromagnetic shunt (2), a winding (3), a winding frame (4), a core (5), a housing (6), permanent magnets (7) located along the inner perimeter of the housing, and a base (8). In the design of a bistable electromagnet, a non-magnetic rod, which is connected to the movable contacts through the shaft levers, also connects the upper and lower armatures, which ensures synchronization of the movement of the armatures when the electromagnet is triggered (not shown in Fig. 2). The main differences of these electromagnets are as follows:

- in a bistable electromagnet, armature fixation in two extreme positions is provided by permanent magnets, and in a monostable one - by permanent magnets (on) and a spring (off);

- a change in the position of the armature in a bistable electromagnet occurs due to a change in the direction of the current in the winding (polarized electromagnet), in a monostable one - by applying voltage to the additional winding.

The design and operation principle of a monostable electromagnet (Fig. 2,a) are considered in [6, 7]. The disadvantages of its design include:

- the charge voltage of the capacitor of the control winding circuit of a monostable electromagnet is $380-400 \mathrm{~V}$, which is unsafe for maintenance personnel;
- significant (in relation to the dimensions of the circuit breaker) dimensions;

- the presence of two windings (on and off);

- a significant amount of permanent magnets, which increases the cost of the electromagnet.

These shortcomings are the reason for the development of a new design of a bistable polarized electromagnet (Fig. 2,b) [8].

Thus, the goal of the paper is to create a mathematical multiphysics model for calculating the dynamic parameters of a bistable electromagnet based on highly coercive permanent magnets with improved characteristics for medium voltage vacuum circuit breakers. In addition, in the course of experimental studies, it is necessary to verify the adequacy of the created model.

Problem definition. As mentioned above, a monostable electromagnet has several disadvantages. Therefore, when designing and studying a bistable polarized electromagnet, the following tasks are formulated:

- to reduce the size and mass of the magnetic system;

- to reduce the mass of highly coercive permanent magnets used, which will reduce the cost of the electromagnet;

- to reduce the charge voltage of the capacitor to a practically safe value of 100-110 V, while ensuring reliable operation of the circuit breaker (when the touch voltage decreases from $400 \mathrm{~V}$ to $100 \mathrm{~V}$, the total resistance of the human body along the path «arm-arm» increases by about 2.7 times - from about $1300 \Omega$ to about $3500 \Omega$, and the direct current through the human body decreases by about 11 times - from about $310 \mathrm{~mA}$ to about $28 \mathrm{~mA}$ (see Fig. 2.2 on p. 102 in [1]), which makes a contact scenario almost safe);

- to ensure high thermal stability of the circuit breaker due to the holding force of the bistable electromagnet armature with permanent magnets of at least $6.5 \mathrm{kN}$.

The design shown in Fig. 2,b [8] is obtained on the basis of numerous preliminary calculations and studies. For the final decision on the parameters of the prototype, comparative calculations of the dynamic characteristics of a bistable polarized electromagnet with a monostable one have been carried out. Also, experimental studies of the new actuator have been carried out when it was installed in a vacuum circuit breaker. All calculations are carried out with the same parameters of the opposing force, the mass of contacts, levers and traction insulators, the mechanical work of the forces opposing the movement (the same armature stroke and contact failure) and the specific characteristics of permanent magnets (coercive force and residual magnetic flux density).

The multiphysics model of the actuator given in $[6,9]$ is based on the Maxwell equations and written in terms of the magnetic vector potential for a nonlinear conducting moving medium taking into account the field of permanent highly coercive magnets with boundary conditions - the zero value of the magnetic vector potential at the remote boundary and the axial component of the field on the axis of symmetry. The problem is solved by the Finite Element Method in axisymmetrical formulation, and for discretization in time domain the implicit multistep Adams-Moulton method of the 4th order with adaptive selection of time step values is used. The PDE system solved to obtain electromagnetic field distribution is supplemented by a separate system of nonlinear equations of the discharge circuit of the storage 
capacitor and a system of nonlinear equations of motion $[6,9]$, the form of which is determined by the electrical circuit for connecting the winding to the energy source and the kinematics of the mechanical system under consideration. In the calculations, all moving masses and the opposing force are reduced to the armature of the electromagnet. Since the calculation is carried out in the COMSOL Multiphysics code, the assumptions made during the calculation are minimal: the frictional forces in the bearings (axes) and the change in the resistance of the winding as a result of heating due to their smallness are not taken into account. The calculation consists of two stages: static calculation and dynamic one. To calculate the dynamics, the initial data are the results of a static calculation.

The nature of the movement is significantly affected by a change in the reduced mass of the armature (Fig. 3), which is associated with the closure of contacts at the moment of impact. Assuming that the impact is inelastic and «stretched» it in time by $0.4 \mathrm{~ms}$, we can write:

$$
\frac{d(m \cdot v)}{d t} \cdot \overrightarrow{1}_{\mathrm{s}}=\left(\frac{d m}{d t} \cdot v+m \cdot \frac{d v}{d t}\right) \cdot \overrightarrow{1}_{\mathrm{s}}=\left(\frac{d m}{d s} \cdot v^{2}+m \cdot \frac{d v}{d t}\right) \cdot \overrightarrow{1}_{\mathrm{s}},
$$

where $m$ is the mass reduced to armature; $v$ is the velocity of movement; $s$ is the armature displacement; $\overrightarrow{1}_{s}$ is the directional unit vector.

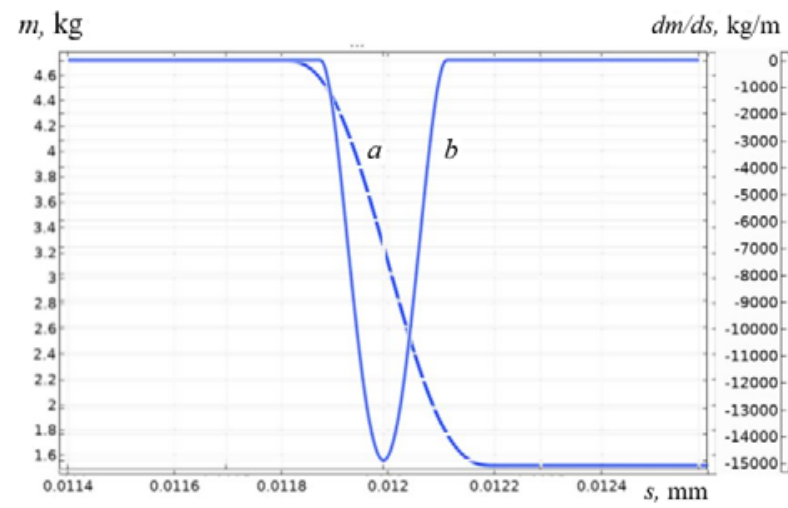

Fig. 3. Change in mass $(a)$ and its derivative $(b)$ as functions of the displacement at the moment of the contacts touch

Table 1 shows the parameters of the windings of the electromagnets and the supply circuit. The initial voltage at the capacitor $U_{c 0}$ is chosen as the minimum voltage for the operation of electromagnets.

Table 1

Parameters of electromagnet windings and supply circuit

\begin{tabular}{|c|c|c|}
\hline Parameter & Monostable & Bistable \\
\hline$U_{\mathrm{c} 0}, \mathrm{~V}$ & 400 & 105 \\
\hline number of turns $w$ & 700 & 270 \\
\hline$R, \Omega$ & 19 & 1.53 \\
\hline$C, \mu \mathrm{F}$ & 10000 & 60000 \\
\hline
\end{tabular}

The results of a comparative calculation of the dynamic characteristics of actuators. The results of calculating the movement of the armature of the electromagnets as a function of time when switched on are shown in Fig. 4, and the results of calculating the driving force (it means the difference between the traction force developed by the electromagnets and the opposing force) as a function of time - in Fig. 5 .

From the graphs it follows: the increase in the total force of a monostable electromagnet occurs more quickly, which, combined with a slightly lower mass of the armature and a smaller time constant determines its higher velocity. The graphs show a significant decrease in the driving force at the moment of the contacts touch.

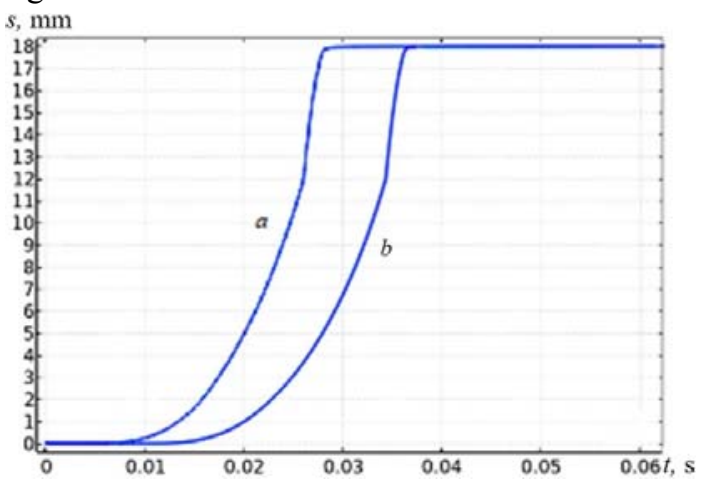

Fig. 4. Displacement of the armature of monostable (a) and bistable (b) electromagnets as a function of time

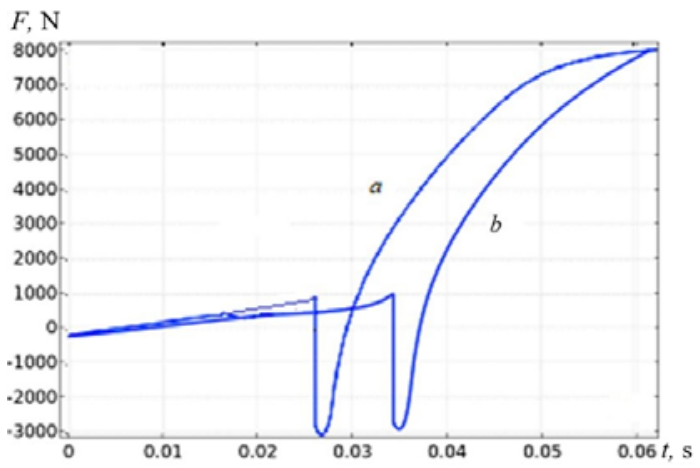

Fig. 5. Comparative characteristics of the driving force $F$ created by monostable $(a)$ and bistable $(b)$ electromagnets

The influence of changes in the reduced mass during the movement of the system is illustrated by the velocity graphs (Fig. 6), which show its jump at the moment of contact closure. In this case, the kinetic energy of the remaining moving parts of the actuator (armature, levers, contact holder) increases which should be enough to overcome the area in which the driving force is negative. Otherwise, the system may «freeze».

It is advisable to carry out a further comparative analysis by the magnetizing force of the windings, which is shown in Fig. 7. The winding currents are presented in Fig. 8.

With almost the same magnetizing force of the windings (the opposing characteristic is the same), the currents of electromagnets vary significantly due to different winding data (windings sizes, wire diameter, number of turns, etc.).

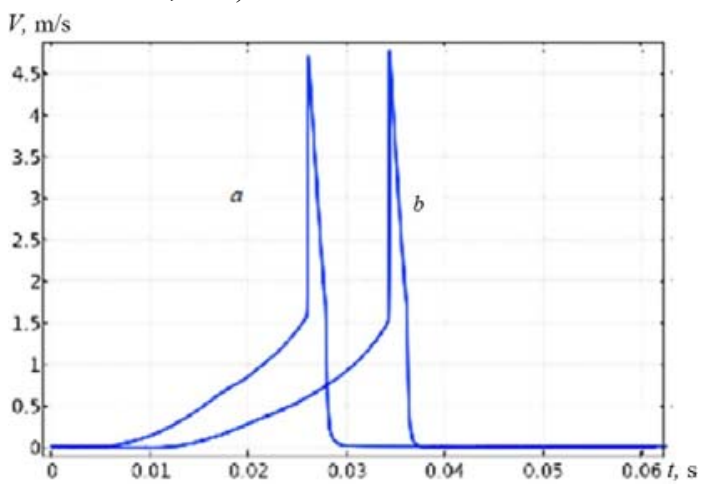

Fig. 6. Velocity of the armature movement of the monostable $(a)$ and bistable $(b)$ electromagnets 
It should be noted that the graphs of currents allow to evaluate the actuator operating parameters [4].

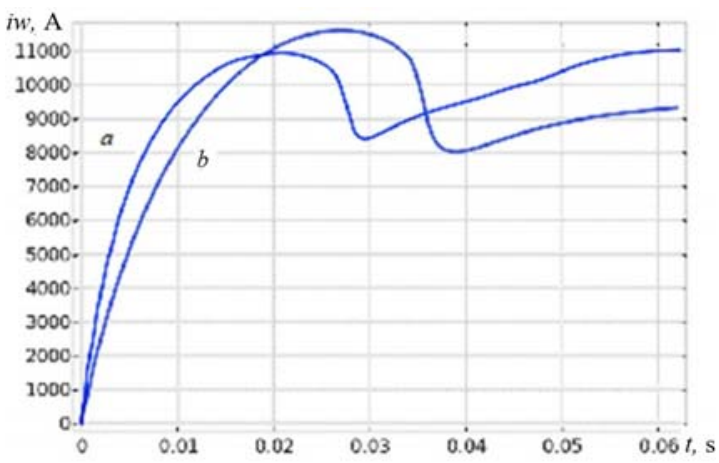

Fig. 7. Magnetizing force of the monostable $(a)$ and bistable $(b)$ electromagnets

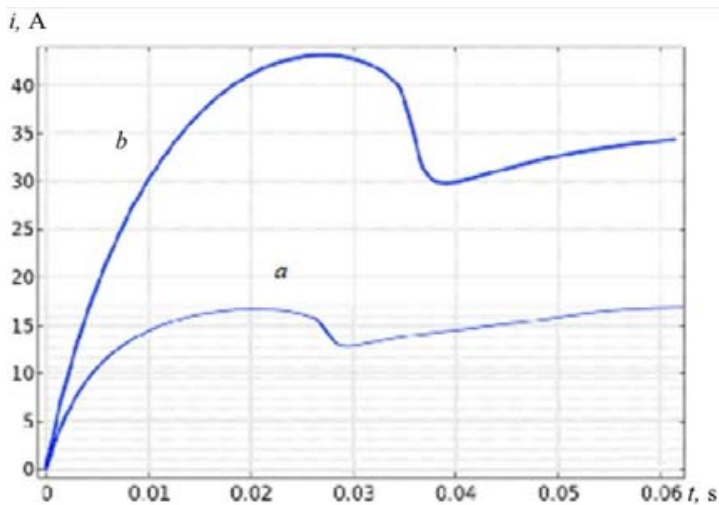

Fig. 8. Currents of windings of the monostable $(a)$ and bistable $(b)$ electromagnets

The minimum of the current curve approximately corresponds to the actuator operating time, and the current fluctuation in the winding circuit means a possible «freezing» of the drive mechanism, which is unacceptable, since it causes the circuit breaker contacts to not switch on completely, leading to an accident. Such a case is shown in Fig. 9 (solid line is the touch line of the contacts) when the capacitor charge voltage $U_{c 0}$ in calculation is insufficient for the actuator to operate.

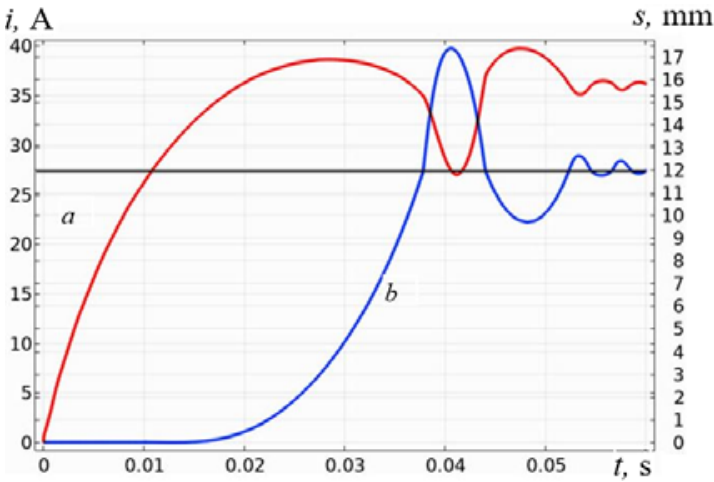

Fig. 9. Current and stroke of the armature during emergency switching on of the electromagnet:

$a$-winding current; $b$ - electromagnet armature stroke

From Fig. 9 it follows that before reaching its final position, the electromagnet armature, levers, traction insulators and contacts started the reverse movement and passed the touch point of the contacts in the opposite direction. Then the contacts opened, the opposing force decreased (see Fig. 1), and the contacts again began to move in the direction of closure, «hanging» at the point of contact. This means that when short circuit current is switched on, the contacts and vacuum chambers can be damaged as a result of burning on the contacts of a powerful electric arc. Closing the contacts of the circuit breaker with small contact pressure is a serious accident, significantly reducing the thermal stability of the circuit breaker both during the flow of rated and emergency currents.

Since the function of switching an electric circuit by a circuit breaker assumes both connecting consumers to energy sources and disconnecting them from these sources, the next step is to study the process of disconnecting a load by a circuit breaker. Figure 10 shows the time dependence of the armature displacement of a new bistable electromagnet during switching off.

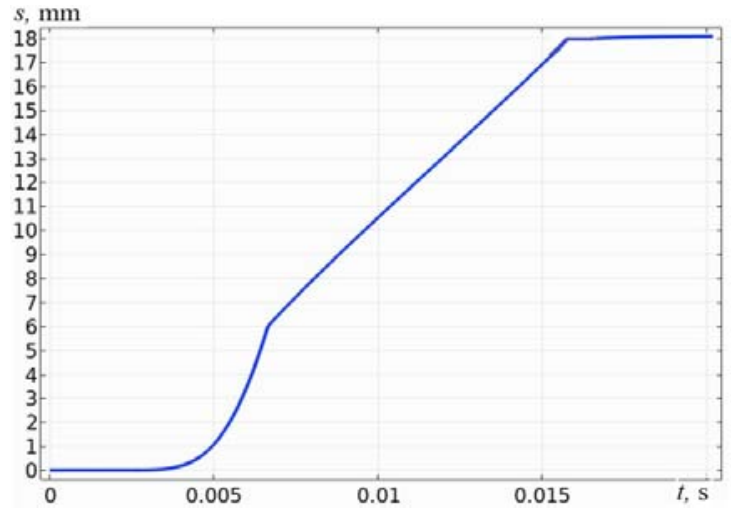

Fig. 10. Dependence of the displacement of the armature of the electromagnet during switching off

An analysis of this dependence shows that the breaker contacts open in about $7 \mathrm{~ms}$, and the off process takes place within $16-17 \mathrm{~ms}$ which is approximately two times faster than switching on (Fig. 4,b and Fig. 10).

Of interest is also the form of the total force acting on the armature of the electromagnet which is shown in Fig. 11.

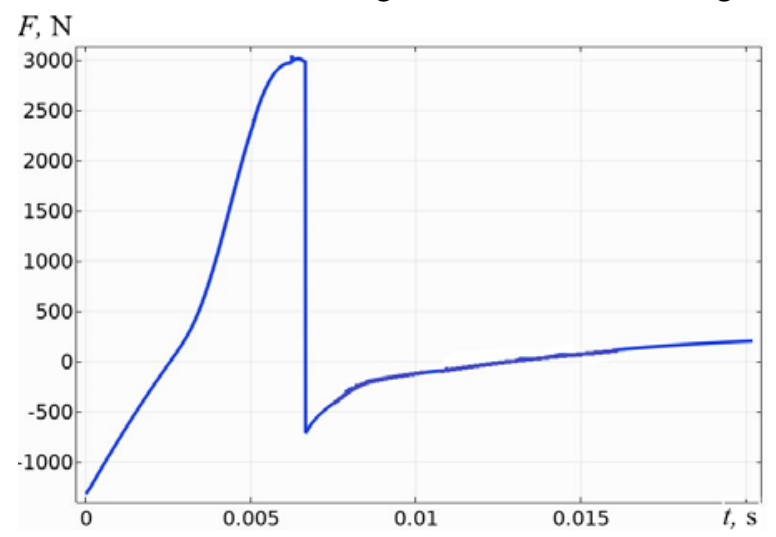

Fig. 11. The total force acting on the armature of an electromagnet when switched off as a function of time

The velocity graph is shown in Fig. 12. As follows from the graph, at the moment of opening the contacts, the velocity of the system decreases due to the attachment to the armature of the mass of contacts, levers and traction insulators.

Indicative is the graph of the current in the winding circuit, which, due to the counter-EMF of armature movement, takes negative values, i.e. recharges the capacitor (see Fig. 13). 


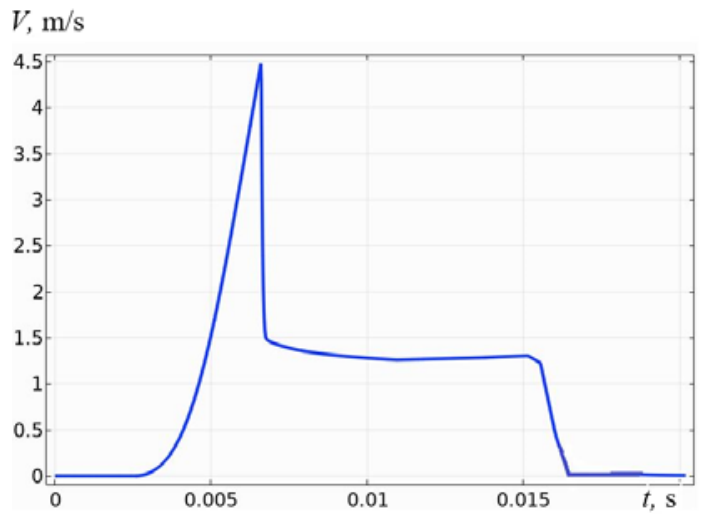

Fig. 12. The electromagnet armature velocity during switching off

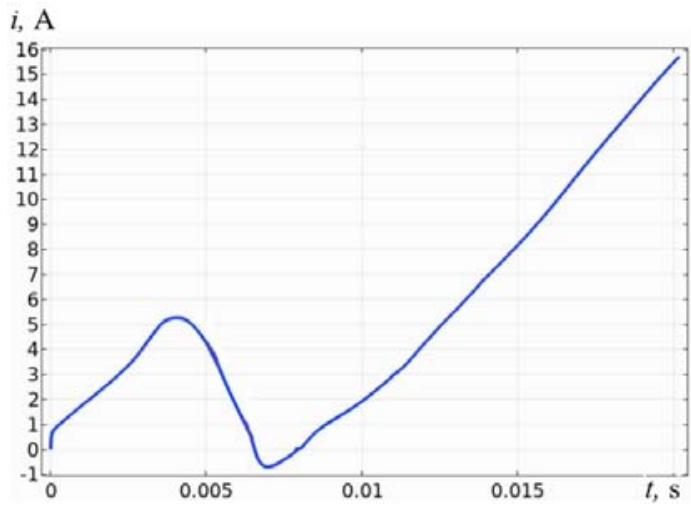

Fig. 13. Winding current change during switching off

This follows from the fact that, according to Ohm law

$$
i=\frac{U_{\mathrm{c}}+E}{r}
$$

where $U_{\mathrm{c}}$ is the voltage on the capacitor; $E$ is the counterEMF of movement; $r$ is the resistance of the winding and connecting wires.

But at the same time

$$
E=-w \cdot \frac{d \Phi}{d t}=-w \cdot \frac{d \Phi}{d s} \cdot v,
$$

where $w$ is the number of turns; $\Phi$ is the magnetic flux; $s$ is the displacement of the armature; $v$ is the velocity.

Therefore, if the velocity is high, then the counterEMF can be larger in magnitude than the voltage on the capacitor. In this case, the current will be negative.

It should be noted that due to the opposing springs, the magnetizing force sufficient to switch off is approximately $1.5 \mathrm{kA}$, while to switch on is about $10 \mathrm{kA}$. Figure 14 shows the nature of the change in voltage on the capacitor during switching off.

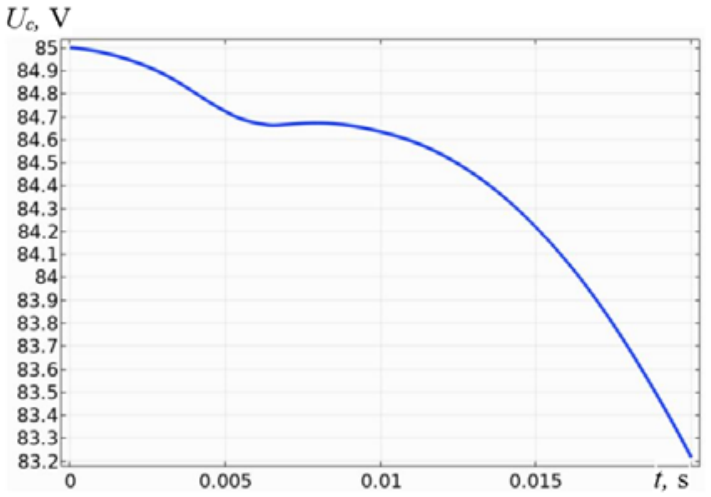

Fig. 14. Capacitor voltage change during switching off
As follows from Fig. 14, due to the large capacitance, the voltage during the switching off time of about16 ms varies slightly (by less than $1 \mathrm{~V}$ ), and the decrease in voltage during the total switch on duration is about $20 \mathrm{~V}$ ).

This gives grounds to assert that the standard operation OFF-ON-OFF will be successfully performed provided that the energy source (capacitor) is disconnected from the electromagnet winding in a timely manner using, for example, the position sensor when the circuit breaker changes its state.

An experimental study of the developed design of a bistable electromagnet. For experimental studies, a prototype bistable electromagnet has been manufactured, which is installed in the casing of a vacuum circuit breaker. A capacitor bank is used as the power source of the electromagnet, and the electric circuit is closed to the winding of the electromagnet and is not turned off until the capacitor bank is completely discharged.

Figure 15 shows the oscillogram of the switching on of the electromagnet at $U_{c 0}=105 \mathrm{~V}$.

Figure 16 shows the oscillogram of the switching off of the electromagnet at $U_{c 0}=60 \mathrm{~V}$.

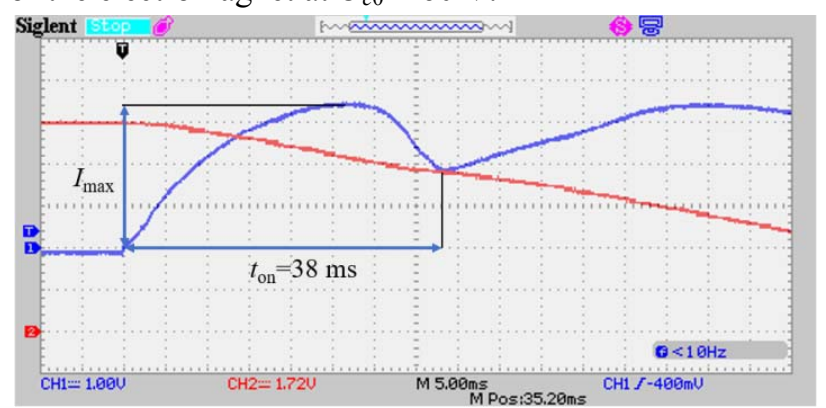

Fig. 15. The oscillogram of the switching on of a bistable actuator, where the horizontal time scale is $5 \mathrm{~ms} / \mathrm{div}$; the vertical current scale is $10 \mathrm{~A} / \mathrm{div}$

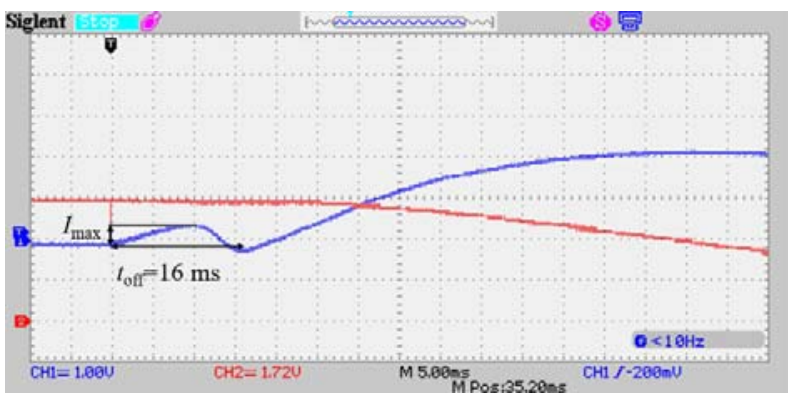

Fig. 16. The oscillogram of the switching off of a bistable actuator, where the horizontal time scale is $5 \mathrm{~ms} / \mathrm{div}$; the vertical current scale is $10 \mathrm{~A} / \mathrm{div}$

A comparative analysis of the calculated and experimental temporal characteristics of the bistable actuator is shown in Table 2, where $I_{\max }$ is the first maximum of the current of the electromagnet winding.

Table 2

Comparative analysis of calculated and experimental temporal characteristics of a bistable actuator

\begin{tabular}{|c|c|c|c|c|}
\hline & \multicolumn{2}{|c|}{ Switching on } & \multicolumn{2}{c|}{ Switching off } \\
\hline & Calculation & Experiment & Calculation & Experiment \\
\hline$I_{\max }, \mathrm{A}$ & 39.0 & 43.0 & 3.6 & 4.0 \\
\hline$t, \mathrm{~ms}$ & 38.0 & 38.0 & 9.5 & 16.0 \\
\hline
\end{tabular}

The discrepancy between the experimental and calculated data (especially in time during the switching off process) can be for various reasons, the most likely of which are the following: 
- uncertainty of the electrical resistivity of the material of the magnetic circuit;

- parameters of permanent magnets;

- friction forces in hinges and bearings;

- change in contact failure due to repeated switching operations, etc.

The uncertainty of the electrical resistivity of the material of the magnetic circuit is the dominant factor, which is confirmed by the results of studies in [10].

During testing, with insufficient capacitor charge voltage, the electromagnet did not switch on completely, at which the armature became «stuck» at the value of the failure. The oscillogram of this process is shown in Fig. 17, which confirms the data obtained by calculation (see Fig. 9).

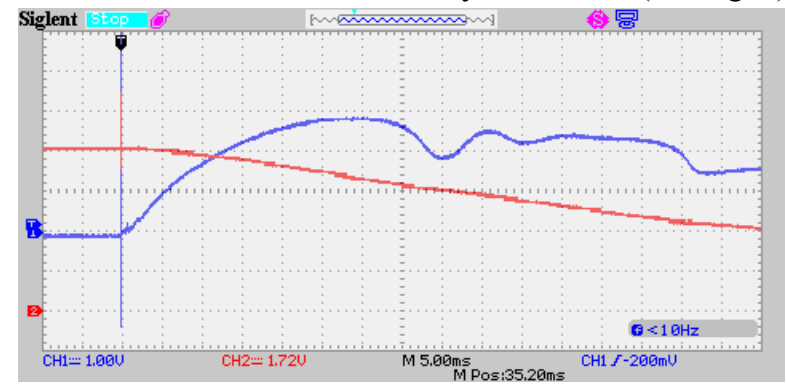

Fig. 17. The process of fuzzy switching on of an electromagnet

Comparative mass and dimensional characteristics of monostable and developed bistable electromagnets are presented in Table 3.

Table 3

Comparative mass and dimensional characteristics of electromagnets

\begin{tabular}{|c|c|c|}
\hline & Monostable & Bistable \\
\hline Diameter, mm & 150 & 140 \\
\hline Height, mm & 110 & 90 \\
\hline Electromagnet mass, kg & 11.5 & 8.63 \\
\hline Copper mass, kg & 0.44 & 0.73 \\
\hline Permanent magnets mass, kg & 0.49 & 0.33 \\
\hline
\end{tabular}

\section{Conclusions.}

1. The created mathematical multiphysics model allows to determine the basic dynamic parameters of a bistable electromagnet: switching on and switching off times, armature velocity, etc.

2. The developed design of a bistable electromagnet, compared with the known monostable design, has outer diameter smaller by $6.7 \%$, lower by $18.2 \%$ height, which, in this case, is a decisive factor for a limited space inside the housing of the vacuum circuit breaker. The mass of the new electromagnet is also decreased by $25 \%$.

3 . The analysis of static traction characteristics with deenergized windings in the drawn position of the armature gives the following values: the well-known monostable electromagnet - $8.5 \mathrm{kN}$; a new developed bistable electromagnet $-6.9 \mathrm{kN}$ with the required $6.5 \mathrm{kN}$. The force of $8.5 \mathrm{kN}$ is unreasonably high for this type of circuit breaker. Such a load requires strengthening of the circuit breaker housing, drive rods and shaft. From this point of view, the developed bistable electromagnet is more preferable.

4. The ratio of the holding force to the total mass of the electromagnet in the new design is $800 \mathrm{~N} / \mathrm{kg}$, while for a monostable electromagnet this indicator is $740 \mathrm{~N} / \mathrm{kg}$. At the same time, the mass of the permanent magnets decreased by $32.6 \%$.

5 . Since the switching off time of the actuators is mainly determined by the force of the opposing springs, the difference in switching off time is insignificant. The switching off time is $16 \mathrm{~ms}$, and the switching on time is $37 \mathrm{~ms}$, which is comparable to the switching on time of the vacuum circuit breakers manufactured by ABB. Therefore, the result obtained for the developed bistable electromagnet is completely acceptable.

6 . The results of investigations allow to talk about the prospects of using the developed design of the electromagnet as an actuator of medium voltage vacuum circuit breakers.

\section{REFERENCES}

1. Klymenko B.V. Elektrychni aparaty. Zahalnyi kurs [Electric apparatus. The general course]. Kharkiv, Tochka Publ., 2013. 400 p. (Ukr).

2. VM1-T. Vacuum circuit-breaker. Available at: https://library.e.abb.com/public/e9408d38c8324932ac60aa9e935 e481a/MA VM1-T(EN)- 1VCD601412\%20-\%20BA\%20543-

02.pdf (accessed 06 April 2019).

3. Klymenko B.V., Bugaychuk V.M., Grechko A.M. A preproduction model of two-position electromagnetic drive for an average-voltage vacuum switch. Electrical engineering \& electromechanics, 2005, no. 2, pp. 23-27. (Rus).

4. Baida E.I. Fuzzy dynamic response of a bistable electromagnetic actuator based on high-coercitivity permanent magnets. Electrical engineering \& electromechanics, 2012, no. 5, pp. 18-20. (Rus).

5. Ro J.-S., Hong S.-K., Jung H.-K. Characteristic analysis and design of a novel permanent magnetic actuator for a vacuum circuit breaker. IET Electric Power Applications, 2013, vol. 7, no. 2, pp. 87-96. doi: 10.1049/iet-epa.2012.0176.

6. Bayda Ye.I., Vyrovets S.V. Characteristic of monostable actuator starting. Naukovyi Visnyk Natsionalnoho Hirnychoho Universytetu, 2013, issue 6, pp. 72-77. (Rus).

7. Klymenko B.V., Vyrovets S.V., Forkun Ya.B. Elektromagnitnyj pryvid [Electromagnetic actuator]. Patent UA, no. 15210,2006 . (Ukr)

8. Bugaychuk V.M., Klymenko B.V., Varshamova I.S., Grechko O.M. Elektromagnitnyj pryvid [Electromagnetic actuator]. Patent UA, no. 130401, 2018. (Ukr).

9. Baida E.I. A mathematical model for calculating the dynamics of on-off electromagnetic actuators of medium voltage vacuum circuit breakers. Journal of scientific publications of graduate students and doctoral students, 2013, no. 1, pp. 136-141. (Rus).

10. Grechko A.M. Experimental research of eddy currents in the magnetic core on dynamic characteristics of the electromagnetic actuator, Bulletin of NTU «KhPI». Series: Problems of Electrical Machines and Apparatus Perfection. The Theory and Practice, 2012, no. 49 (955), pp. 9-17. (Rus).

Received 30.04.2020

E.I. Baida ${ }^{1}$,Doctor of Technical Science, Associate Professor, B.V. Klymenko, Doctor of Technical Science, Professor, S.V. Vyrovets ${ }^{1}$, Senior Lecturer,

M.G. Pantelyat ${ }^{1}$, Candidate of Physics and Mathematics, Associate Professor,

M. Clemens ${ }^{2}$, Professor, Dr. rer. nat.

${ }^{1}$ National Technical University «Kharkiv Polytechnic Institute»,

2, Kyrpychova Str., Kharkiv, 61002, Ukraine,

e-mail: baida.kpi@gmail.com,vvs_2007@ukr.net,

m150462@yahoo.com

${ }^{2}$ University of Wuppertal,

Rainer-Gruenter-Straße 21, 42119 Wuppertal, Germany,

e-mail: clemens@uni-wuppertal.de

How to cite this article:

Baida E.I., Klymenko B.V., Vyrovets S.V., Pantelyat M.G., Clemens M. Investigations of the dynamics of a bistable electromagnet with improved characteristics for medium voltage vacuum circuit breakers. Electrical engineering \& electromechanics, 2020, no. 3, pp. 3-8. doi: 10.20998/2074-272X.2020.3.01. 